\title{
Tumor-infiltrating CD8+ T cells combined with tumor-associated CD68+ macrophages predict postoperative prognosis and adjuvant chemotherapy benefit in resected gastric cancer
}

Jun $\mathrm{Lu}^{1,2,3+}, \mathrm{Yu} X \mathrm{Xu}^{4,5+}$, Yuan Wu $\mathrm{u}^{4,5}$, Xiao-yan Huang ${ }^{1,2,3}$, Jian-wei Xie ${ }^{1,2,3}$, Jia-bin Wang ${ }^{1,2,3}$, Jian-xian Lin ${ }^{1,2,3}$, Ping Li $i^{1,2,3}$, Chao-hui Zheng ${ }^{1,2,3^{*}}$, Ai-min Huang ${ }^{4,5^{*}}$ and Chang-ming Huang ${ }^{1,2,3^{*}}$ (D)

\begin{abstract}
Background: Tumor-infiltrating immune cells are present in various malignant tumors, but their clinical significance in gastric cancer (GC) remains unclear. This study aimed to investigate the prognostic significance of tumor-infiltrating lymphocytes (TILs) and tumor-associated macrophages (TAMs).

Methods: Using a prospective database containing 401 cases of GC, we evaluated TIL (cluster of differentiation 8 (CD8) expression) and TAM (cluster of differentiation 68 (CD68) expression) statuses via immunohistochemical staining.

Results: Compared with CD8+ TIL-negative cases $(n=196,48.6 \%)$, CD8+ TIL-positive cases $(n=205,51.1 \%)$ showed significantly better recurrence-free survival (RFS) [log-rank $p<0.001$; multivariate HR: 0.372; 95\% confidence interval (Cl): 0.239-0.579, $p<0.001]$. In contrast, compared with CD68+ TAM-negative cases $(n=217,54.1 \%)$, CD68+ TAM-positive cases $(n=184,45.9 \%)$ had significantly poor RFS [log-rank $p<0.001$; multivariate HR: 2.182; 95\% Cl: 1.435-3.318, $p<0.001$ ]. Thus, patients with a positive CD8+ TIL and negative CD68+ TAM status exhibited significantly increased RFS. Multivariate analysis demonstrated that CD8+ TILs and CD68+ TAMs may serve as independent prognostic markers for RFS. Incorporating CD8+ TIL and CD68+ TAM statuses into the AJCC TNM system generated a predictive model with better predictive accuracy for RFS. More importantly, patients with a positive TIL and negative TAM status showed a tendency of improved RFS after postoperative adjuvant chemotherapy (PAC). Similar results were obtained by overall survival (OS) analysis.
\end{abstract}

Conclusions: CD8+ TIL and CD68+ TAM statuses were identified as independent prognostic factors that may be integrated into the current TNM staging system to refine risk stratification and to better predict the survival benefit from PAC in patients with GC.

(Continued on next page)

\footnotetext{
*Correspondence: wwkzch@163.com; draimin@163.com; hcmlr2002@163.com

'Jun Lu and Yu Xu contributed equally to this work and should be considered co-first authors.

'Department of Gastric Surgery, Fujian Medical University Union Hospital, Fuzhou, China

${ }^{4}$ Department of Pathology, the School of Basic Medical Sciences, Fujian

Medical University, Fuzhou, China

Full list of author information is available at the end of the article
}

(c) The Author(s). 2019 Open Access This article is distributed under the terms of the Creative Commons Attribution 4.0 International License (http://creativecommons.org/licenses/by/4.0/), which permits unrestricted use, distribution, and reproduction in any medium, provided you give appropriate credit to the original author(s) and the source, provide a link to the Creative Commons license, and indicate if changes were made. The Creative Commons Public Domain Dedication waiver (http://creativecommons.org/publicdomain/zero/1.0/) applies to the data made available in this article, unless otherwise stated. 
(Continued from previous page)

Trial registration: The current controlled trial was registered at ClinicalTrials.gov (ID: NCT02327481) on December 30, 2014.

Keywords: Adjuvant chemotherapy benefit, Gastric cancer, Prognosis, Tumor-infiltrating neutrophils, Tumor-associated macrophages

\section{Background}

Gastric cancer (GC) is one of the most common malignant tumors in both sexes worldwide [1]. Regarding advanced GC, it has been suggested that a PAC regimen based on 5-fluorouracil (5-Fu) should be used as a firstline postoperative therapy [2-6]. However, the response to PAC varies among patients. Of greater concern is the lack of a reliable criterion for identifying patients at high risk of recurrence, which in turn makes it difficult to distinguish between patients who will benefit from PAC when considering the survival rate after surgery [6-8]. Accordingly, there is considerable interest in exploring the potential benefits of PAC for GC patients.

In recent decades, convincing evidence has emerged that the tumor microenvironment (TME) and inflammation play a key role in the development of many malignant tumors, including GC $[1,9,10]$. Tumor-infiltrating lymphocytes (TILs) and tumor-associated macrophages (TAMs) are two main components of the TME that have shown prognostic value in previous studies [11, 12]. Therefore, incorporating these immunological parameters into the established TNM staging system may increase the prognostic value for further stratification and better management of patients with different prognoses.

Regardless, little is known about the prognostic significance of TILs combined with TAMs in GC, and the impact of these cells on PAC benefit in GC remains unclear. In this study, we evaluated TILs and TAMs in more than $400 \mathrm{GC}$ patients by immunohistochemical staining of CD8 and CD68. In addition, we used subgroup analysis to explore the relationship between TILs/TAMs and survival rates as well as the benefit of PAC based on 5-FU for survival. The results of this study will clarify the important prognostic role of lymphocyte and macrophage infiltration in GC and help to redefine subgroups of patients who are likely to benefit from PAC.

\section{Methods}

\section{Patients and specimens}

From January 1, 2015, to April 1, 2016, 438 patients were recruited from Union Medical College Hospital of Fujian Medical University for a randomized clinical trial. The final analysis included 419 patients (clinicaltrials.gov No. NCT02327481). Detailed information on the selection, exclusion, quality control and randomization has been reported previously $[13,14]$. This study is a substudy of the above clinical trials. After excluding 10 patients with neuroendocrine cancer, 6 patients with palliative surgery and 2 patients without GC evidence, this analysis involved 401 patients who underwent therapeutic gastrectomy and pathologically confirmed as having stage I, II, or III gastric adenocarcinoma (pT1-4aN0-3 M0) according to the 7th American Joint Committee on Cancer staging [15]. The formalin-fixed paraffin-embedded (FFPE) tissues of the 401 patients with GC were analyzed, with all specimens being independently re-evaluated by two gastrointestinal pathologists. None of the patients had received any anticancer treatment before surgery. PAC treatment is acceptable for patients with advanced or early diagnosis of excessive lymph node metastasis, and PAC was classified as received or not received [6]. A total of 256 patients $(63.8 \%)$ received at least one 5 -FU baseline PAC cycle $[8,16]$. According to the Japanese Classification of Gastric Carcinoma, PAC with S-1 is recommended for GC patients with pathological stage II or III disease [17]. In our center, fluoride-assisted chemotherapy is also recommended for most stage II or III GC patients, depending on the patient's wishes and physical condition [18]. In this cohort, 256 patients (63.8\%) received 5-FU adjuvant chemotherapy with a median cycle of 5 (range 1-12), similar to a previous study [19]. Specifically, 76.2\% (195/ 256) received the SOX regimen (S-1 plus oxaliplatin), $12.1 \%(31 / 256)$ the FOLFOX regimen (5-FU + oxaplatin+leucovorin), 9.4\% (24/256) the S1 + paclitaxel regimen, and $2.3 \%(6 / 256)$ the $\mathrm{S} 1+$ docetaxel regimen. Overall survival (OS) was defined as the interval between the date of surgery and the date of death or last visit. Recurrencefree survival (RFS) was defined as the period from the date of surgery to the date of diagnosis of recurrence. The median follow-up time was 29 months (range 3-41 months).

All patients provided written informed consent before sampling, and the research procedure was approved by the Institutional Review Committee of Union Medical College Hospital of Fujian Medical University.

\section{Immunohistochemistry (IHC) and evaluation}

Formalin-fixed, paraffin-embedded GC surgical specimens were used for IHC, as previously described [20, 21]. Briefly, continuous paraffin slices with a thickness of $4 \mu \mathrm{m}$ were immersed in xylene and rehydrated by an ethanol series, PBS buffer and deionized water for 
5 min each. The slides were heated to $100^{\circ} \mathrm{C}$ for 20 min in $\mathrm{pH} 9$ Tris-based solution. All slides were incubated with the primary antibody for $60 \mathrm{~min}$ at $37^{\circ} \mathrm{C}$ for $1 \mathrm{~h}$ (dilutions: rabbit anti-human CD8 monoclonal 1:500, SP16, MAIXIN. BIO, Fuzhou, China; mouse anti-human CD68 monoclonal 1:500, KP1, MAIXIN. BIO, Fuzhou, China) and then washed. As a secondary antibody, mouse IgG was added for $30 \mathrm{~min}$, and the slides were again washed. The sections were processed with the universal SP Elivision-plus kit (kit9903, MAIXIN. BIO, Fuzhou, China), and the sections were counterstained with hematoxylin.

All specimens were selected by the local pathologist. Two pathologists (Y. X and AM. H) blinded to the tumor clinicopathologic characteristics and patient outcomes independently scored all slides, with discrepancies resolved by consensus. The tissue sections were screened in a low-power field $(100 \times)$, and the 5 most representative fields were selected using an Olympus BX46 research microscope (Olympus, Tokyo, Japan).

CD8 is mainly distributed on the cell membrane and in cytoplasm. Using high-power microscopy, 5 fields of view with the richest permeability of GC were selected from each slice. The percentage of CD8+ T lymphocytes among total lymphocytes was calculated. The average values of 5 fields were taken as the density (\%) of CD8 + TILs. Briefly, TILs were counted separately based on their location in the epithelium or interstitium. T lymphocytes infiltrating into cancer cell nests, designated intraepithelial $\mathrm{T}$ lymphocytes, were counted in high-power fields (HPFs) at 200x magnification. Intratumoral T cells were classified as 0,1 , 2 , or 3 ( $\leq 5,6$ to 19,20 to 34 , or $\geq 35$ T cells per highpower field, respectively), and the average count was calculated. Stromal (tumor-associated stroma and/or perivascular spaces) $\mathrm{T}$ lymphocytes were evaluated by the same method. To assess the density of tissue-infiltrating CD68+ TAMs, the respective areas of the tumor nest and peritumoral tissue were measured at $200 \times$ magnification. The number of nucleated CD68+ TAMs in each area was then counted manually, and the results are expressed as cells per field. Positive-staining cells less than or equal to the size of circulating monocytes $(\sim 10 \mu \mathrm{m})$ were excluded. CD68+ TAMs were scored as $0,1,2$, or $3(\leq 10,11$ to 29 , 30 to 49 , or $\geq 50$ TAMs/ high-power field, respectively). The average number of the two researchers was used in the following analysis to minimize variability. After hematoxylin-eosin staining, the presence or absence of fibrous capsules was assessed using the same serial sections from the same blocks as used for CD68 immunohistochemistry. Representative pictures are provided in Additional file 1: Figure S1.

The semi-quantitative immunohistochemical grading of TILs and TAMs in tumors was determined by highpower microscopy (when there were more than five regions, the most abundant lymphocyte infiltration area was selected by the "hot spot" method) [22, 23]. Briefly, five fields with the richest infiltration of $\mathrm{GC}$ were selected from each slice, and the percentages of TILs and TAMs were calculated. The average of five fields was used as the density of TILs and TAMs [24]. First, a quantitative score based on the estimated percentage of immunopositive-stained cells among total cells was specified according to the following scale: $1(<1 \%$ cells); 2 ( $1-10 \%$ cells); 3 (11-33\% cells); 4 (34-66\% cells); and 5 (67-100\% cells). Immunopositive cells were defined as those showing partial or complete staining within the cytoplasm and/or plasma membrane. Second, staining intensity was scored as follows: 0 (none), $1+$ (mild), 2+ (moderate), and 3+ (intense). Finally, scores (ranging from 1 to 8 ) were calculated by adding the percentage positivity scores and the intensity scores for each section. The patient cohort was divided into two groups using the median value, with negative or positive CD8+ or CD68+ expression.

\section{Statistical analysis}

Correlation between clinicopathological features and immunohistochemical variables was evaluated by the Chi-square test or Fisher exact test, as appropriate. The Kaplan-Meier method was employed to estimate the survival curve and the log-rank test for survival analysis. Important factors identified by univariate analysis were examined in multivariate analysis. A Cox proportional hazard model was applied for multivariate analysis to evaluate the independent effects of variables. The Harrell index of concordance (C-index), Akaike information criterion (AIC) [16], and Bayesian information criterion (BIC) [25] were calculated to compare the accuracy of the prognostic models. In addition, decision curve analysis was performed to determine the clinical utility of the prognostic model [26]. The statistical analyses were performed using SPSS 19.0 (SPSS Inc., Chicago, IL) and $\mathrm{R}$ version 3.1.2 (R Foundation for Statistical Computing, Vienna, Austria). Statistical significance was set at a 2 -sided $p<0.05$.

\section{Results \\ Association of CD8+ TIL and CD68+ TAM with clinicopathological features}

The patient clinicopathological features are summarized in Table 1. In this study, there were $401 \mathrm{pa}$ tients [271 male (67.6\%), 130 female (32.4\%)]. Positive expression of CD8+ TIL and CD68+ TAM were $51.1 \%$ (205 out of 401$)$ and $45.9 \%$ (184 out of 401 ) in our set, respectively. A CD8+ TIL-positive status negatively correlated with lymphovascular invasion 
Table 1 Correlation between TIL, TAM and clinicopathologic characteristics in the 401 gastric cancers

\begin{tabular}{|c|c|c|c|c|c|c|}
\hline \multirow[t]{2}{*}{ Characteristics } & \multicolumn{2}{|l|}{ CD8+ TIL } & \multirow{2}{*}{$\begin{array}{l}p \\
\text { value }\end{array}$} & \multicolumn{2}{|l|}{ CD68+ TAM } & \multirow{2}{*}{$\begin{array}{l}p \\
\text { value }\end{array}$} \\
\hline & Negative (\%) & Positive (\%) & & Negative (\%) & Positive (\%) & \\
\hline All cases & $196(48.6)$ & $205(51.1)$ & & $217(54.1)$ & $184(45.9)$ & \\
\hline Sex & & & 0.596 & & & \\
\hline Female & $61(31.1)$ & 69 (33.7) & & $72(33.2)$ & $58(31.5)$ & 0.749 \\
\hline Male & 135 (68.9) & $136(68.3)$ & & $145(66.8)$ & $126(68.5)$ & \\
\hline Age (y) & & & 0.915 & & & 0.668 \\
\hline$<65$ & $134(68.4)$ & $139(67.8)$ & & $150(69.1)$ & $123(66.8)$ & \\
\hline$\geq 65$ & 62 (31.6) & $66(32.2)$ & & 67 (30.9) & 61 (33.2) & \\
\hline Tumor location & & & 0.805 & & & 0.330 \\
\hline Proximal & $58(29.6)$ & $59(28.8)$ & & 68 (31.3) & 49 (26.6) & \\
\hline Middle & $30(15.3)$ & $39(19.0)$ & & $37(17.1)$ & $32(17.4)$ & \\
\hline Distal & $96(49.0)$ & $95(46.3)$ & & $103(47.5)$ & 88 (47.8) & \\
\hline Entire & $12(6.1)$ & $12(5.9)$ & & $9(4.1)$ & $15(8.2)$ & \\
\hline Tumor size $(\mathrm{cm})$ & & & 0.260 & & & 0.441 \\
\hline$<5$ & $114(58.2)$ & $131(63.9)$ & & 137 (63.1) & $108(58.7)$ & \\
\hline$\geq 5$ & $82(41.8)$ & $74(36.1)$ & & $80(36.9)$ & $76(41.3)$ & \\
\hline Differentiation & & & 0.858 & & & 0.840 \\
\hline Differentiated & $83(42.3)$ & 85 (41.5) & & $92(42.4)$ & $76(41.3)$ & \\
\hline Undifferentiated & $113(57.7)$ & $120(58.5)$ & & 125 (57.6) & $108(58.7)$ & \\
\hline Lymphovascular invasion & & & 0.044 & & & 0.130 \\
\hline Absent & $101(51.5)$ & $127(62.0)$ & & $131(60.4)$ & $97(52.7)$ & \\
\hline Present & $95(48.5)$ & $78(38.0)$ & & 86 (39.6) & $87(47.3)$ & \\
\hline Tumor depth & & & 0.158 & & & 0.225 \\
\hline $\mathrm{T} 1 / 2$ & $75(38.3)$ & $93(45.4)$ & & $97(44.7)$ & 71 (38.6) & \\
\hline $\mathrm{T} 3 / 4$ & $121(61.7)$ & $112(54.6)$ & & $120(55.3)$ & $113(61.4)$ & \\
\hline Lymph node metastasis & & & 0.014 & & & 0.919 \\
\hline Absent & $65(33.2)$ & $93(45.4)$ & & 86 (39.6) & $72(39.1)$ & \\
\hline Present & $131(66.8)$ & $112(54.6)$ & & $131(60.4)$ & $112(60.9)$ & \\
\hline Pathological stage & & & 0.123 & & & 0.561 \\
\hline 1 & $57(29.1)$ & $78(38.0)$ & & 78 (35.9) & $57(31.0)$ & \\
\hline$\|$ & $41(20.9)$ & $43(21.0)$ & & $43(19.8)$ & $41(22.3)$ & \\
\hline III & $98(50.0)$ & $84(41.0)$ & & $96(44.2)$ & $86(46.7)$ & \\
\hline Adjuvant chemotherapy & & & 0.222 & & & 0.917 \\
\hline Absent & $65(33.2)$ & $80(39.0)$ & & 79 (36.4) & 66 (35.9) & \\
\hline Present & $131(66.8)$ & $125(61.0)$ & & 138 (63.6) & $118(64.1)$ & \\
\hline
\end{tabular}

$(p=0.044)$ and lymph node metastasis $(p=0.014)$, whereas such a correlation was not found in the CD68+ TAM-positive subgroup. Moreover, CD8+ TIL and CD68+ TAM did not significantly correlate with tumor size $(p=0.260, p=0.441)$, tumor differentiation $(p=0.858, \quad p=0.840)$, tumor depth $(p=0.158, \quad p=$ $0.225)$ or TNM stage $(p=0.123, p=0.561)$. No significant correlations were found between expression of CD8+ TIL or CD68+ TAM and adjuvant chemotherapy. (Table 1).

\section{Correlation between the CD8+ TIL/CD68+ TAM status and} prognosis

Patients who were CD8+ TIL positive showed significantly improved RFS (Additional file 2: Figure S2A; $p<$ 0.001 ) and OS (Additional file 2: Figure S2C; $p<0.001$ ) compared with CD8+ TIL-negative patients. As show by Additional file 2: Figure S2B and Additional file 2: Figure S2D, the RFS and OS of the CD68+ TAM-positive group were significantly lower than that of the CD68+ TAMnegative group (both $p<0.001$ ). 
Univariate and multivariate regression analysis

Lymphovascular involvement $(p=0.009)$, TNM stage $(p<$ 0.001 ), adjuvant chemotherapy (HR: 0.577, $p=0.039$ ), positive CD8+ TIL status (HR: $0.372, p<0.001$ ) and positive CD68+ TAM status $(p<0.001)$ were identified as independent prognostic factors that associated with RFS (Additional file 5: Table S1). Other factors including sex, age, tumor location, tumor size, tumor differentiation, were not significantly associated with patient recurrencefree survival. Similar results were obtained in the OS analysis (Additional file 5: Table S2).

\section{The combination of CD8+ TIL and CD68+ TAM statuses}

We classified the gastric cancer cases into 4 groups based on their CD8+ TIL and CD68+ TAM statuses (Fig. 1). The proportion of the patients in groups $1,2,3$, and 4 was $28.9 \% \quad(n=116), 22.2 \% \quad(n=89), 25.2 \% \quad(n=101)$, and $23.7 \%$ ( $n=95)$, respectively. Interestingly, we found no significant differences in RFS and OS between group 2 and group 3 ( $p=0.633, p=0.899$, respectively, Fig. 1a, c). Thus, we defined group 1 as type I, groups 2 and 3 as type II, and group 4 as type III (Fig. 1b, d). Additional stratified analysis showed significant differences in RFS and OS among these 3 types in the stage II/III cohort (Fig. 1e-h). However, no differences were observed in RFS and OS among these 3 types for stage I disease due to the excellent prognosis of stage I patients (data not shown).

\section{Extension of the TNM staging system to include CD8+ TIL/CD68+ TAM status}

When analyzing RFS, the C-index was 0.7699, when assessed only with TNM stage, and the outcomes improved to 0.8126, when CD8+ TIL/CD68+ TAM status was added. Also in the RFS analysis, the AIC and BIC were 1009.875 and 1013.869, respectively, when assessed only with TNM stage, and the outcomes decreased to 998.756 and 1001.750, respectively, when CD8+ TIL/CD68+ TAM status was added (Table 2). Similar results were obtained from OS analysis which corroborated the results from the RFS analysis (Table 2).

\section{Assessment of the clinical utility of the prognostic model with CD8+ TIL/CD68+ TAM status using decision curve analysis}

Next, we assessed the clinical application of the prognostic model that included CD8+ TIL/CD68+ TAM status. The results indicate that the constructed model is advantageous with a higher threshold probability and improved performance for predicting 3-year RFS and 3-year OS than the TNM stage or CD8+ TIL/CD68+ TAM status alone (Additional file 3: Figure S3).

\section{Associations between postoperative adjuvant chemotherapy (PAC) and the CD8+ TIL/CD68+ TAM status} For the entire cohort of patients who did not receive PAC treatment, the CD8+ TIL/CD68+ TAM status was not associated with OS (Additional file 4: Figure S4B; $p=0.085)$. In contrast, for stage II/III patients who did not receive PAC, the CD8+ TIL/CD68+ TAM status was not associated with RFS or OS (Fig. $2 \mathrm{a} ; p=0.126$ and Fig. $2 b, p=0.126$, respectively). Importantly, both in the entire cohort and in stage II/III patients who received PAC, those that were classified as type I had better RFS

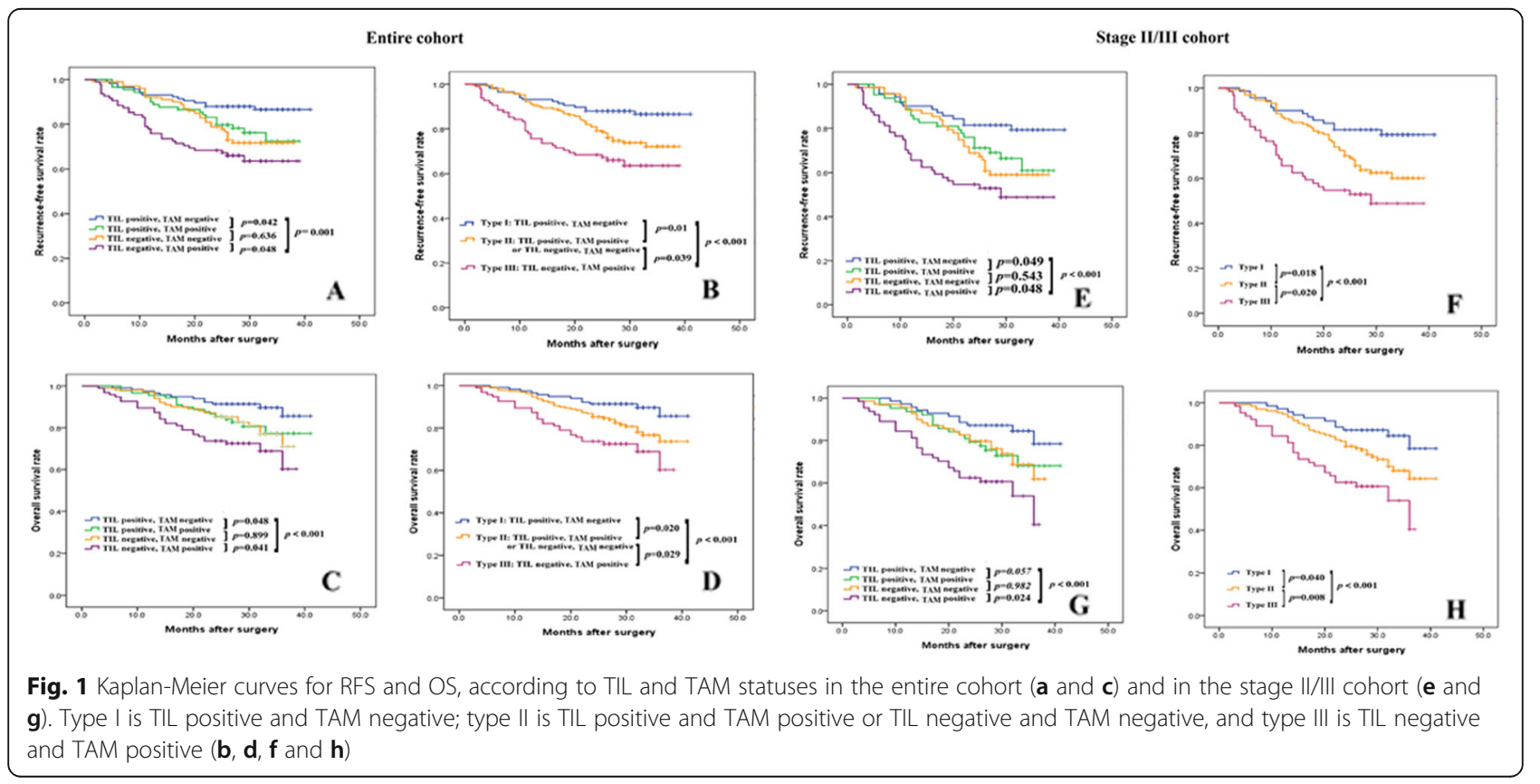


Table 2 Comparison of the prognostic accuracies of TNM staging system and tumour-infiltrating immune cells

\begin{tabular}{|c|c|c|c|c|}
\hline \multirow[t]{2}{*}{ RFS } & \multicolumn{3}{|l|}{ Model } & \multirow{2}{*}{$\begin{array}{l}p \\
\text { value }\end{array}$} \\
\hline & TNM & TIL + TAM & TNM + (TIL + TAM) & \\
\hline C-index (95\% Cl) & $0.7699(0.7159-0.8013)$ & $0.6055(0.5538-0.6782)$ & $0.8126(0.7791-0.8504)$ & $<0.001$ \\
\hline AIC & 1009.875 & 1104.745 & 998.756 & I \\
\hline $\mathrm{BIC}$ & 1013.869 & 1108.739 & 1001.750 & I \\
\hline \multicolumn{5}{|l|}{ OS } \\
\hline C-index (95\% Cl) & $0.7428(0.7053-0.7932)$ & $0.6329(0.5881-0.7011)$ & $0.8030(0.7626-0.8485)$ & $<0.001$ \\
\hline AIC & 825.9906 & 880.5326 & 815.0896 & I \\
\hline $\mathrm{BIC}$ & 829.9846 & 884.5266 & 820.0835 & / \\
\hline
\end{tabular}

C-index indicates Harrell concordance index; AIC indicates Akaike Information Criterion, BIC indicates Bayesian Information Criterion, AUC indicates area under the curve

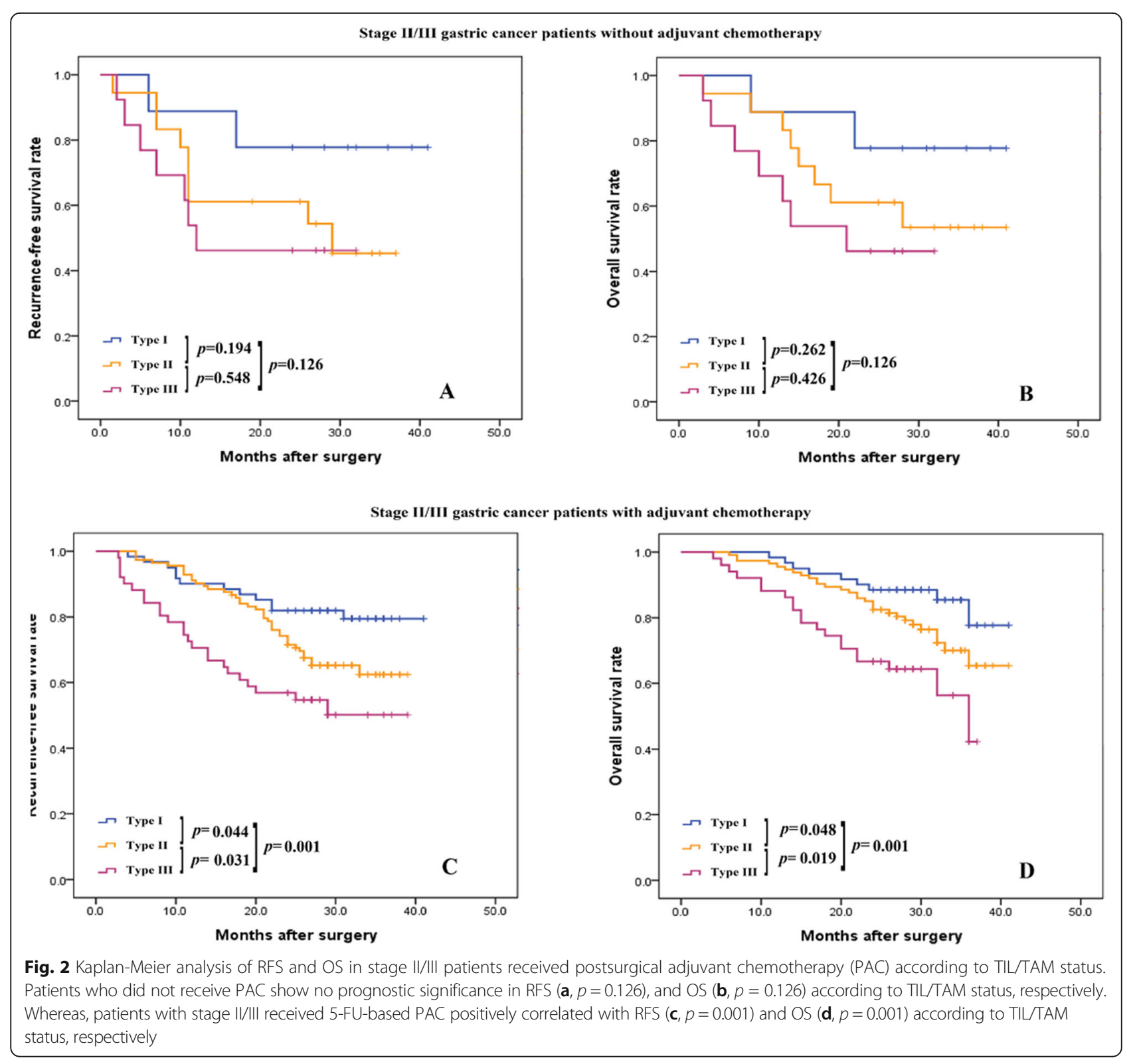


and OS than those classified as type II or III (Additional file 4: Figure S4C, $p<0.001$, Additional file 4: Figure S4D, $p<0.001$; and Fig. 2c, $p=0.001$, Fig. 2D, $p=0.001$, respectively). Patients who received PAC and were CD8+ TIL positive and CD68+ TAM negative had a reduced risk of short survival, both in the entire cohort and in the subgroup with stage II/III disease (HR: 0.466, 95\% CI: $0.265-0.821, p=0.008$; HR: 0.458 , 95\% CI: $0.257-$ $0.817, p=0.008$, respectively), whereas patients who did not receive PAC did not show the same risk benefit (Table 3). Altogether, these results suggest that gastric cancer patients with a CD8+ TIL-positive and CD68+ TAM-negative status (defined as type I above) might benefit more from PAC.

\section{Discussion}

GC is an inflammation-related cancer characterized by a large degree of multinuclear and monocyte infiltration, including lymphocytes and macrophages [16, 27]. Tumor-associated immune cells affect complex microenvironments, with prognostic value in previous studies $[11,16,28]$. To date, there are several markers for TILs, such as CD3, CD4, CD8, Foxp3, and Granzyme B [29]. In the present study, we stained for $\mathrm{CD} 8$ on $\mathrm{T}$ cells, as reported [24,30]. TAMs can be classified into two phenotypes: M1 (classically activated macrophages) and M2 (alternatively activated macrophages) [31, 32], and using immunochemistry, TAMs can be assessed by anti-CD68, M1-type TAMs by anti-HLA-DR and M2-type TAMs by anti-CD163 [32, 33]. However, it is largely unclear which macrophages have the greatest impact on the efficacy of chemotherapy for GC [34]. CD68 has been widely accepted as a specific marker of TAMs in human cancer [35, 36]. A recent report found high expression of CD68+ TAMs to be associated with recurrence of melanoma [36], and other studies have reported the clinical and functional significance of CD68+ TAMs in GC, though without addressing the M1 and M2 subsets [37, 38]. Thus, we also used CD68 as a marker of TAMs in this study.
Previous studies have explored the relationship between TIL infiltration and GC outcomes, but these studies have produced different results $[39,40]$. For example, Fukuda et al. [39] found no significant difference in survival rates among patients with significant or slight TIL infiltration. In contrast, Lee et al. reported that the OS of GC patients with a high CD8+ TIL density tended to be longer than that of patients with a low TIL density at the same TNM stage, which was consistent with our findings [40]. In our study, the RFS and OS of TIL-positive cases were significantly better than those of TIL-negative cases. These conflicting results may be caused by different methods used to determine TIL strength. In addition, the function of intratumoral CD8+ lymphocytes in tumors is interlinked with other immune cells, and accumulating evidence suggests that TAMs plays an important role in cancer progression [41-43]. However, conflicting prognostic data have been reported [33]. Ishigami et al. [44] observed a negative correlation between TAMs and the prognosis of GC patients, whereas Ohno et al. [45] concluded that the aggregation of TAMs within the tumor nest had a beneficial effect. Zhang et al. [46] also evaluated TAMs in 180 GC patients and found no significant correlation between these cells and OS. In the present study, CD68+ TAMs were found to be an independent risk factor for a worse $\mathrm{GC}$ prognosis.

Indeed, it is difficult to characterize complex tumor microenvironment using a single immune marker [43]. Nakanishi et al. reported that the ratio of CD68+ macrophages/CD57+ cells was closely related to prognosis of renal cell carcinoma [47]. Therefore, the main purpose of this study was to explore the effect of a combination of TILs and TAMs on the prognosis and efficacy of chemotherapy for GC, rather than the effect of different types of macrophages on prognosis. In fact, the use of multiparametric analysis to characterize immune cell types, such as CD8+ TILs and CD68+ TAMs, in the present study may provide a more comprehensive picture of the immune phenotypes within the TME, which may help in stratifying patients with the best chance of

Table 3 Hazard ratios for risk of mortality in gastric cancer patients receiving postoperative adjuvant chemotherapy (pac) or not according to TIL and TAM status

\begin{tabular}{|c|c|c|c|c|}
\hline & \multirow[b]{2}{*}{ Variable } & \multicolumn{3}{|l|}{ Risk of mortality } \\
\hline & & No. of patients & (TIL positive + TAM & \\
\hline \multirow[t]{3}{*}{ Entire cohort } & & 401 (100\%) & $\mathrm{HR}(95 \% \mathrm{Cl})$ & $p$ value \\
\hline & With PAC & $256(63.8 \%)$ & $0.466(0.265-0.821)$ & 0.008 \\
\hline & Without PAC & $145(36.2 \%)$ & $0.543(0.195-1.515)$ & 0.243 \\
\hline \multirow[t]{3}{*}{ Stage II/II cohort } & & $266(100 \%)$ & & \\
\hline & With PAC & $226(85.0 \%)$ & $0.458(0.257-0.817)$ & 0.008 \\
\hline & Without PAC & 40 (15.0\%) & $0.534(0.191-1.496)$ & 0.233 \\
\hline
\end{tabular}


responding to immunotherapy. Using the CD8+ TIL/ CD68+ TAM status, we were able to classify samples into 3 types (Type 1: CD8+ TIL positive and CD68+ TAM negative; Type 2: CD8+ TIL positive and CD68+ TAM positive or CD8+ TIL negative and CD68+ TAM negative; Type 3: CD8+ TIL negative and CD68+ TAM positive).

Traditional prognostic models for GC patients depend on the TNM staging system, which is derived from biological phenotypes centered on cancer cells. In fact, even patients with the same stage of cancer may have very different prognoses. In this study, we first found that incorporating CD8+ TILs/CD68+ TAMs into the current TNM staging system may increase prognostic value, thereby better identifying patients with different prognoses and providing better risk-oriented treatment. In general, incorporating molecular biomarkers or biologically driven classification into staging systems may better predict prognosis and treatment options and promote the development of personalized or precise medicine $[48,49]$. TILs and TAMs are key in tumor-related inflammation and binding with other immune cells, which are processes considered to be evidence of host-growth interaction with tumors [31, 33, 50]. Previously, Zhang et al. generated a nomogram integrating TAMs, tumor $\mathrm{T}$ stage, $\mathrm{N}$ stage, and distant metastasis to predict the OS rate of GC [46]. In this study, we generated a novel and powerful staging system for GC patients based on TIL/TAM-based immune status and the tumor cell-centered TNM system. Our results suggest that incorporating the TIL/TAM status into the established TNM system can more accurately quantify prognostic risk.

Patients with stage II or III GC are considered to be candidates for PAC. However, it is uncertain whether all patients need PAC, as a large proportion of them do not appear to benefit from this approach. Therefore, the development of an improved PAC benefit prediction model has gained much interest [6]. Indeed, there is much evidence that the success of anticancer therapies, including traditional cytotoxic compounds, radiotherapy and targeted drugs, depends, at least in part, on activation of the anticancer immune response [51]. As previously described, CD68+ TAMs increase the response of many malignant tumors (such as stage III colorectal cancer) to 5-FU adjuvant therapy [52]. In this study, we further assessed the relationship between TILs/TAMs and survival in a subgroup of stage II/III patients receiving PAC. The results indicated that among patients receiving PAC, those with simultaneously high TIL and low TAM infiltration were more likely to have increased RFS and OS rates compared with the other 3 groups, indicating that CD8+ TILs/CD68+ TAMs may be an important factor in predicting the effect of chemotherapy. Immunotherapy, including immune checkpoint blockade, has been considered an important component of anticancer therapy [53, 54], and the TIL/TAM status might be an interesting target of investigation for the immunotherapy options that are emerging in this setting. A recent study found that PD-L1 is not only expressed in cancer cells but also in TILs at a high rate [55]. Furthermore, Harada et al. reported that TAMs are highly associated with PD-L1 expression in GC cells, suggesting that macrophage infiltration is a potential therapeutic target [56]. The authors also indicated that CSF1/CSF1R blockade might reduce PD-L1 expression in tumor cells [56]. Clinically, the status of CD8+ TILs/ CD68+ TAMs is helpful for stratifying patients with stage II/III GC receiving PAC, and prognostic stratification based on the TIL/TAM status can guide doctors in adopting tailormade treatment plans according to patient performance. For example, strengthening a 5-FU-based treatment regimen or adding macrophage-targeted therapies (such as CSF1/CSF1R and/or PD-L1 inhibitors) is a potential strategy for type II or III GC with good performance. Currently, a phase 1, open-label, global study is underway to assess the combination of CSF1R antagonists and checkpoint blockades (NCT0323191). These results may help clinicians better select patients who need more aggressive adjuvant therapy or more in-depth follow-up, even though these patients might not be considered to be at high risk according to traditional clinicopathological features.

This study has some limitations. First, the study was retrospective. Although all parameters of prospective clinical trials were included, the number of patients receiving PAC was relatively small, and the prognostic significance of the PAC regimen and PAC cycle was not compared. Second, as a currently unresolved issue [24, 57], the specimens used and semi-quantitative immunohistochemical evaluation may not fully reflect the status of the tumor immune microenvironment. TAMs are more diverse and heterogeneous than cells with single markers or M1/M2 phenotypes. Further studies are needed to determine the interaction between different TAM and cancer outcomes. Third, the follow-up time was relatively short. Overall, in-depth in vitro and in vivo experiments are urgently needed to provide mechanical insight.

\section{Conclusions}

The TIL/TAM status is a promising biomarker for predicting the prognosis of GC. Our model incorporates immune parameters into the established TNM staging system to help clinicians and patients quantify the benefits of adjuvant chemotherapy after GC resection and formulate personalized treatment recommendations and treatment decisions. However, these results should be validated by a large, prospective, multiagency study. 


\section{Additional files}

Additional file 1: Figure S1. TIL and TAM status in gastric cancer. A, TIL status in the invasive margin of tumors. Original magnification, 200x (left panels). B, TAM status in the invasive margin of tumors. Original magnification, 200× (right panels). (TIF $331 \mathrm{~kb}$ )

Additional file 2: Figure S2. Kaplan-Meier curves for RFS and OS of gastric cancer patients according TIL ( $A$ and $C$ ) or TAM (B and D) status. (TIF $271 \mathrm{~kb}$ )

Additional file 3: Figure S3. The TNM staging system combined with TIL/TAM status was compared to the TNM staging system alone and TIL/ TAM status alone in terms of 3-year RFS and OS (A and B). Using decision curve analysis, the TNM staging system combined with TIL/TAM status showed superior net benefit compared to the TNM staging system alone and TIL/TAM status alone. (TIFF 304 kb)

Additional file 4: Figure S4. Kaplan-Meier analysis of RFS and OS in entire cohort patients received postsurgical adjuvant chemotherapy (PAC) according to TIL/TAM status. Patients who did not receive PAC show prognostic significance in RFS (,$p=0.046)$, but no prognostic significance in OS ( $B, p=0.085$ ) according to TIL/TAM status, respectively. Whereas, patients received 5-FU-based PAC positively correlated with RFS $(C, p<0.001)$ and OS (D, $p<0.001)$ according to TIL/TAM status, respectively. (TIF $346 \mathrm{~kb}$ )

Additional file 5: Table S1. Univariate and Multivariable Analysis of Recurrence-free Survival in 401 Patients With Gastric Cancer. Table S2. Univariate and Multivariable Analysis of Overall Survival in 401 Patients With Gastric Cancer. (DOCX 21 kb)

\section{Abbreviations}

CD68: Cluster of differentiation 68; CD8: Cluster of differentiation 8; GC: Gastric cancer; OS: Overall survival; PAC: Postoperative adjuvant chemotherapy; RFS: Recurrence-free survival; TAM: Tumor-associated macrophages; TIL: Tumor-infiltrating lymphocytes

\section{Acknowledgements}

We thank Bin-bin Xu, Yun Dai and Zhi-fang Zheng for their assistance provided in patient screening and data input.

\section{Authors' contributions}

$J \mathrm{~L}, \mathrm{YX}, \mathrm{AMH}, \mathrm{CHZ}$, and $\mathrm{CMH}$ conceived the study, analyzed the data, and drafted the manuscript; $Y W, X Y H, P L$, and JWX helped revise the manuscript critically for important intellectual content; JBW and JXL helped collect data and design the study. All authors have read and approved the final manuscript.

\section{Funding}

This study was supported by the National Nature Science Foundation of China (No. 81871899). Construction Project of Fujian Province Minimally Invasive Medical Center (No. [2017]171). The second batch of special support funds for Fujian Province innovation and entrepreneurship talents (2016 B013). QIHANG funds of Fujian Medical University (No. 2016QH025). Natural Science Foundation of Fujian Province (2019 J01155). These funding bodies had no role in the design of the study and collection, analysis, interpretation of data and in writing the manuscript.

\section{Availability of data and materials}

The datasets used and/or analyzed during the current study are available from the corresponding author on reasonable request.

\section{Ethics approval and consent to participate}

The study protocol conformed to the ethical guidelines of the 1975 Declaration of Helsinki, and the Ethics Committee of Fujian Medical University Union Hospital. Written informed consent was obtained before resection.

\section{Consent for publication}

Not applicable.

\section{Competing interests}

The authors declare that they have no competing interests.

\section{Author details}

${ }^{1}$ Department of Gastric Surgery, Fujian Medical University Union Hospital, Fuzhou, China. ${ }^{2}$ Department of General Surgery, Fujian Medical University Union Hospital, Fuzhou, China. ${ }^{3}$ Key Laboratory of Ministry of Education of Gastrointestinal Cancer, Fujian Medical University, Fuzhou, China.

${ }^{4}$ Department of Pathology, the School of Basic Medical Sciences, Fujian Medical University, Fuzhou, China. ${ }^{5}$ Institue of Oncology of Fujian Medical University, Fuzhou, China.

Received: 4 February 2019 Accepted: 26 August 2019

Published online: 14 September 2019

\section{References}

1. Siegel RL, Miller KD, Jemal A. Cancer statistics, 2016. CA Cancer J Clin. 2016;1:7-30.

2. De Vita F, Orditura M, Matano E, et al. A phase II study of biweekly Oxaliplatin plus Infusional 5-fluorouracil and Folinic acid (FOLFOX-4) as first-line treatment of advanced gastric Cancer patients. Br J Cancer. 2005;9:1644-9.

3. Sakuramoto S, Sasako M, Yamaguchi T, et al. Adjuvant chemotherapy for gastric Cancer with S-1, an Oral Fluoropyrimidine. N Engl J Med. 2007;18: 1810-20.

4. Sasako M, Sakuramoto $\mathrm{S}$, Katai $H$, et al. Five-year outcomes of a randomized phase III trial comparing adjuvant chemotherapy with S-1 versus surgery alone in stage II or III gastric Cancer. J Clin Oncol. 2011;33:4387-93.

5. Tsuburaya A, Yoshida K, Kobayashi M, et al. Sequential paclitaxel followed by Tegafur and uracil (UFT) or S-1 versus UFT or S-1 monotherapy as adjuvant chemotherapy for T4a/b gastric Cancer (SAMIT): a phase 3 factorial randomised controlled trial. Lancet Oncol. 2014:8:886-93.

6. Jiang Y, Li T, Liang X, et al. Association of Adjuvant Chemotherapy with survival in patients with stage II or III gastric Cancer. Jama Surg. 2017;7:e171087.

7. Lim L, Michael M, Mann GB, Leong T. Adjuvant therapy in gastric Cancer. J Clin Oncol. 2005;25:6220-32.

8. Wu S, He H, Liu H, et al. C-C motif chemokine 22 predicts postoperative prognosis and adjuvant chemotherapeutic benefits in patients with stage II/ III gastric Cancer. Oncoimmunology. 2018;6:e1433517.

9. Mantovani A. Cancer: Inflaming Metastasis. Nature. 2009;7225:36-7.

10. Hanahan D, Weinberg RA. Hallmarks of Cancer: the next generation. Cell. 2011;5:646-74.

11. Galon J, Costes A, Sanchez-Cabo F, et al. Type, density, and location of immune cells within human colorectal tumors predict clinical outcome. Science. 2006:5795:1960-4.

12. Wiktor-Jedrzejczak W, Gordon S. Cytokine regulation of the macrophage (M phi) system studied using the Colony stimulating Factor-1-deficient Op/Op mouse. Physiol Rev. 1996:4:927-47.

13. Zheng $\mathrm{CH}$, Lu J, Zheng HL, et al. Comparison of 3D laparoscopic gastrectomy with a 2D procedure for gastric Cancer: a phase 3 randomized controlled trial. Surgery. 2018;2:300-4.

14. Lu J, Zheng $\mathrm{CH}$, Zheng $\mathrm{HL}$, et al. Randomized, controlled trial comparing clinical outcomes of 3D and 2D laparoscopic surgery for gastric Cancer: an interim report. Surg Endosc. 2017;7:2939-45.

15. Washington K. 7th edition of the AJCC Cancer staging manual: stomach. Ann Surg Oncol. 2010;12:3077-9.

16. Zhang $\mathrm{H}$, Liu H, Shen $\mathrm{Z}$, et al. Tumor-infiltrating neutrophils is prognostic and predictive for postoperative adjuvant chemotherapy benefit in patients with gastric Cancer. Ann Surg. 2018;2:311-8.

17. Sano T, Aiko T. New Japanese classifications and treatment guidelines for gastric Cancer: revision concepts and major revised points. Gastric Cancer. 2011;2:97-100.

18. Lu J, Zheng ZF, Wang W, et al. A novel TNM staging system for gastric Cancer based on the metro-ticket paradigm: a comparative study with the AJCC-TNM staging system. Gastric Cancer. 2019;4:759-68.

19. Chen S, Feng XY, Li YF, Zhao BW, Zhou ZW, Chen YB. The prognosis of gastric Cancer patients with marginally elevated carcinoembryonic antigen (CEA) values after D2 radical gastrectomy. J Surg Oncol. 2013;6:641-5.

20. Chen XJ, Han LF, Wu XG, et al. Clinical significance of CD163+ and CD68+ tumor-associated macrophages in high-risk HPV-related cervical Cancer. J Cancer. 2017;18:3868-75. 
21. Obeid JM, Wages NA, Hu Y, Deacon DH, Slingluff CJ. Heterogeneity of CD8(+) tumor-infiltrating lymphocytes in non-small-cell lung Cancer: impact on patient prognostic assessments and comparison of quantification by different sampling strategies. Cancer Immunol Immunother. 2017:1:33-43.

22. Aghajani MJ, Yang T, McCafferty CE, Graham S, Wu X, Niles N. Predictive relevance of programmed cell death protein 1 and tumor-infiltrating lymphocyte expression in papillary thyroid Cancer. Surgery. 2018;1:130-6.

23. Ding $T, X u J$, Wang $F$, et al. High tumor-infiltrating macrophage density predicts poor prognosis in patients with primary hepatocellular carcinoma after resection. Hum Pathol. 2009:3:381-9.

24. Chen J, He Q, Liu J, et al. CD8+ tumor-infiltrating lymphocytes as a nove prognostic biomarker in lung Sarcomatoid carcinoma, a rare subtype of lung Cancer. Cancer Manag Res. 2018:3505-11.

25. Lu J, Zheng ZF, Xie JW, et al. Is the 8th edition of the AJCC TNM staging system sufficiently reasonable for all patients with noncardia gastric Cancer? A 12,549-patient international database study. Ann Surg Oncol. 2018;7:2002-11.

26. Huang $Y Q$, Liang $C H$, He L, et al. Development and validation of a Radiomics nomogram for preoperative prediction of lymph node metastasis in colorectal Cancer. J Clin Oncol. 2016:18:2157-64.

27. Ishigami S, Natsugoe S, Tokuda K, et al. Prognostic value of Intratumoral natural killer cells in gastric carcinoma. Cancer-Am Cancer Soc. 2000;3:577-83.

28. Mao $Y$, Feng $Q$, Zheng $P$, et al. Low tumor infiltrating mast cell density confers prognostic benefit and reflects Immunoactivation in colorectal Cancer. Int J Cancer. 2018.

29. Lee JY, Son T, Cheong JH, et al. Association between chemotherapyresponse assays and subsets of tumor-infiltrating lymphocytes in gastric Cancer: a pilot study. J Gastric Cancer. 2015;4:223-30.

30. Morihiro T, Kuroda S, Kanaya N, et al. PD-L1 expression combined with microsatellite instability/CD8+ tumor infiltrating lymphocytes as a useful prognostic biomarker in gastric Cancer. Sci Rep. 2019;1:4633.

31. Biswas SK, Allavena P, Mantovani A. Tumor-associated macrophages: functional diversity, clinical significance, and open questions. Semin Immunopathol. 2013;5:585-600

32. Yamaguchi T, Fushida S, Yamamoto $Y$, et al. Tumor-associated macrophages of the M2 phenotype contribute to progression in gastric Cancer with peritoneal dissemination. Gastric Cancer. 2016:4:1052-65.

33. Zhang QW, Liu L, Gong CY, et al. Prognostic significance of tumorassociated macrophages in solid tumor: a meta-analysis of the literature. PLoS One. 2012;12:e50946.

34. Minami K, Hiwatashi K, Ueno S, et al. Prognostic significance of CD68, CD163 and folate receptor-Beta positive macrophages in hepatocellular carcinoma. Exp Ther Med. 2018;5:4465-76.

35. Wang L, Zhang C, Zhang Z, et al. Specific clinical and immune features of CD68 in glioma via 1,024 samples. Cancer Manag Res. 2018:6409-19.

36. Salmi S, Siiskonen H, Sironen R, et al. The number and localization of CD68+ and CD163+ macrophages in different stages of cutaneous melanoma. Melanoma Res. 2019:3:237-47.

37. Zhang J, Yan Y, Yang Y, et al. High Infiltration of Tumor-Associated Macrophages Influences Poor Prognosis in Human Gastric Cancer Patients, Associates with the Phenomenon of EMT. Medicine (Baltimore). 2016;6: e2636.

38. Wu H, XU JB, He YL, et al. Tumor-associated macrophages promote angiogenesis and Lymphangiogenesis of gastric Cancer. J Surg Oncol. 2012;4:462-8

39. Fukuda K, Tsujitani S, Maeta Y, Yamaguchi K, Ikeguchi M, Kaibara N. The expression of RCAS1 and tumor infiltrating lymphocytes in patients with T3 gastric carcinoma. Gastric Cancer. 2002:4:220-7.

40. Lee HE, Chae SW, Lee YJ, et al. Prognostic implications of type and density of tumour-infiltrating lymphocytes in gastric Cancer. Br J Cancer. 2008:10:1704-11.

41. Mantovani A, Sozzani S, Locati M, Allavena P, Sica A. Macrophage polarization: tumor-associated macrophages as a paradigm for polarized M2 mononuclear phagocytes. Trends Immunol. 2002;11:549-55.

42. Li JQ, Yu XJ, Wang YC, et al. Distinct patterns and prognostic values of tumor-infiltrating macrophages in hepatocellular carcinoma and gastric Cancer. J Transl Med. 2017;1:37.

43. Ying L, Yan F, Meng Q, et al. PD-L1 expression is a prognostic factor in subgroups of gastric Cancer patients stratified according to their levels of CD8 and FOXP3 immune markers. Oncoimmunology. 2018;6:e1433520.

44. Ishigami S, Natsugoe S, Tokuda K, et al. Tumor-associated macrophage (TAM) infiltration in gastric Cancer. Anticancer Res. 2003:5A:4079-83.
45. Ohno S, Inagawa $\mathrm{H}$, Dhar DK, et al. The degree of macrophage infiltration into the Cancer cell Nest is a significant predictor of survival in gastric Cancer patients. Anticancer Res. 2003;6D:5015-22.

46. Zhang H, Wang X, Shen Z, Xu J, Qin J, Sun Y. Infiltration of diametrically polarized macrophages predicts overall survival of patients with gastric Cancer after surgical resection. Gastric Cancer. 2015;4:740-50.

47. Nakanishi H, Miyata Y, Mochizuki Y, et al. Pathological significance and prognostic roles of densities of CD57+ cells, CD68+ cells, and mast cells, and their ratios in clear cell renal cell carcinoma. Hum Pathol. 2018:102-8.

48. Tsoukalas N, Tsapakidis K, Kamposioras K. AJCC-8 TNM staging system for gastric Cancer. Is there a scope for improvement? I Investig Surg. 2019:1-2.

49. Lu J, Zheng ZF, Huang CM. ASO Author Reflections: New TNM Classification of Gastric Cancer. Ann Surg Oncol. 2018;(Suppl 3):717-8.

50. Gooden MJ, de Bock GH, Leffers N, Daemen T, Nijman HW. The prognostic influence of tumour-infiltrating lymphocytes in Cancer: a systematic review with meta-analysis. Br J Cancer. 2011;1:93-103.

51. Galluzzi L, Senovilla L, Zitvogel L, Kroemer G. The secret ally: Immunostimulation by anticancer drugs. Nat Rev Drug Discov. 2012;3:215-33.

52. Malesci A, Bianchi P, Celesti G, et al. Tumor-associated macrophages and response to 5-fluorouracil adjuvant therapy in stage III colorectal Cancer. Oncoimmunology. 2017;12:e1342918.

53. Kang BW, Kim JG, Lee IH, Bae HI, Seo AN. Clinical significance of tumorinfiltrating lymphocytes for gastric Cancer in the era of immunology. World I Gastrointest Oncol. 2017;7:293-9.

54. Prestwich R, Vile R, Melcher A. Cancer Immunotherapy. N Engl J Med. 2008; 10:1072, 1073

55. Fang W, Chen Y, Sheng J, et al. Association between PD-L1 expression on tumour-infiltrating lymphocytes and overall survival in patients with gastric Cancer. J Cancer. 2017;9:1579-85.

56. Harada K, Dong X, Estrella JS, et al. Tumor-associated macrophage infiltration is highly associated with PD-L1 expression in gastric adenocarcinoma. Gastric Cancer. 2018;1:31-40.

57. Schalper KA, Brown J, Carvajal-Hausdorf D, et al. Objective measurement and clinical significance of TILs in non-small cell lung Cancer. J Natl Cancer Inst. 2015;3.

\section{Publisher's Note}

Springer Nature remains neutral with regard to jurisdictional claims in published maps and institutional affiliations.

Ready to submit your research? Choose BMC and benefit from:

- fast, convenient online submission

- thorough peer review by experienced researchers in your field

- rapid publication on acceptance

- support for research data, including large and complex data types

- gold Open Access which fosters wider collaboration and increased citations

- maximum visibility for your research: over $100 \mathrm{M}$ website views per year

At $\mathrm{BMC}$, research is always in progress.

Learn more biomedcentral.com/submission 